\title{
LAELIA MOTTAE (OrCHIDACEAE): UNA ESPECIE NUEVA DEL COMPLEJO DE LAELIA ANCEPS LINDL.
}

\author{
Fredy Archila ${ }^{1,2}$, Guy Chiron ${ }^{3}$, Dariusz Szlachetko ${ }^{4}$, Vincenzo Bertolin $I^{5}$ \\ y Eduardo A. Pérez-García ${ }^{6,7}$ \\ 'Estación Experimental de Orquídeas de la Familia Archila, Cobán, A.V., Guatemala \\ ${ }^{2}$ Herbario BIGU, Universidad de San Carlos de Guatemala, Campus Central, Guatemala, Guatemala \\ ${ }^{3}$ Herbiers, Université Claude Bernard Lyon 1, Villeurbanne Cedex, Francia \\ ${ }^{4}$ Department of Plant Taxonomy and Nature Conservation, Gdańsk University, Gdańsk, Polonia \\ ${ }^{5}$ Departamento de Conservación de la Biodiversidad, El Colegio de la Frontera Sur, Tapachula, Chiapas, México \\ ${ }^{6}$ Departamento de Ecología y Recursos Naturales, Facultad de Ciencias, \\ Universidad Nacional Autónoma de México, México D.F., México \\ ${ }^{7}$ Autor para correspondencia: eduardo.perez-garcia@ciencias.unam.mx
}

\begin{abstract}
Resumen: Se describe e ilustra una especie nueva del género Laelia: L. mottae Archila, Chiron, Szlach. \& E.A. Pérez-García. Durante muchos años esta especie se consideró como una variante enana de Laelia anceps; sin embargo, el análisis de ejemplares, tanto silvestres como cultivados, permitió establecer que en realidad se trata de dos especies. Laelia mottae es exclusiva del sureste de Guatemala y del sur de Honduras. Se comparan los principales caracteres morfológicos de L. anceps y L. mottae, y se discuten las diferencias en su distribución, hábitat y cultivo.
\end{abstract}

Palabras clave: Centroamérica, Guatemala, Honduras, Laelia anceps subsp. anceps, Laelia anceps subsp. dawsonii, orquídea.

\begin{abstract}
We describe and illustrate a new species of the genus Laelia: L. mottae Archila, Chiron, Szlach. \& E.A. Pérez-García. For many years, this species was considered a dwarf variant of Laelia anceps; however, the analysis of field-coming and cultivate specimens enabled us to conclude that they actually are two species. Laelia mottae comes from south-eastern Guatemala and southern Honduras. We compare the main morphological characters between L. anceps and L. mottae, and we discuss the differences in distribution, habitat, and cultivation between these two species of horticultural interest.
\end{abstract}

Key words: Central America, Guatemala, Honduras, Laelia anceps subsp. anceps, Laelia anceps subsp. dawsonii, orchid.

A finales del siglo pasado se realizaron algunos avances significativos en la delimitación del género Laelia (van den Berg y Chase, 2000; 2001; 2004a, b; Chiron y Castro-Neto, 2002; van den Berg et al., 2009); no obstante, todavía quedan varios aspectos taxonómicos por atender, como la adecuada circunscripción de las especies y la descripción de algunos taxones nuevos. Este último punto es relevante porque las laelias son un grupo muy importante de orquídeas que presenta una gran interés hortícola; y por lo tanto, se debería esperar que las especies comerciales estuvieran bien clasificadas.

El género Laelia fue descrito por Lindley en 1831, estableciendo como especie tipo a L. grandiflora (La Llave \& Lex.) Lindl. Este taxón se considera actualmente como sinónimo de L. speciosa (Kunth) Schltr. (van den Berg y
Chase, 2004a; Alrich y Higgins, 2008). El grupo de especies más representativo de Laelia se encuentra en las Sierras Madre Occidental, Oriental y Sur, así como en la Faja Volcánica Transmexicana (Halbinger y Soto, 1997; van den Berg et al., 2009), y está formado por L. albida Bateman ex Lindl., L. autumnalis (La Llave \& Lex.) Lindl., L. crawshayana Rchb. f., L. eyermaniana Rchb. f., L. furfuracea Lindl. y $L$. speciosa (Kunth) Schltr. No obstante, existen otras especies del género que se pueden clasificar en tres grupos adicionales, basándose en caracteres de su morfología floral o vegetativa, así como en características ecológicas de las especies que los conforman. El primero de estos tres grupos está formado por Laelia rubescens Lindl. y L. aurea F.Navarro, especies propias de tierras bajas y con pseudobulbos formados por un solo entrenudo. Laelia aurea tiene una distri- 
bución restringida al occidente de México, mientras que $L$. rubescens presenta una amplia distribución geográfica que incluye México, Guatemala, Belice, El Salvador, Honduras, Nicaragua y Panamá (Halbinger y Soto, 1997).

El segundo grupo reconocible incluye a Laelia superbiens Lindl., que presenta plantas morfológicamente muy similares a las del género Schomburgkia, por lo que ha sido ubicada dentro de dicho género con el nombre de $S$. superbiens (Lindl.) Rolfe (van den Berg y Chase, 2004a). Laelia superbiens se conoce de Chiapas, así como de Guatemala, Honduras y Nicaragua (Halbinger y Soto, 1997). Las poblaciones atribuidas a esta especie encontradas en la Sierra Norte de Oaxaca (Salazar et al., 2006) corresponden a un taxón diferente, todavía no descrito (G. Salazar, com. pers.).

El tercero de estos grupos está formado por el complejo de especies de Laelia anceps Lindl., y presenta plantas con características morfológicas intermedias entre las del grupo de L. rubescens y las del de L. superbiens (Halbinger y Soto, 1997). Este carácter transicional tiene soporte en los análisis moleculares (van den Berg y Chase, 2004b). Laelia anceps es una de las especies más cultivadas por los aficionados y quizá por este mismo motivo se han reconocido numerosas variedades de la misma (Halbinger y Soto, 1997). Gran parte de estas variedades corresponden a variantes hortícolas, de forma o de color, de individuos particulares (cultivares) que no son generalizables a los demás individuos de la población, y que por lo tanto no deben considerarse como variedades geográficas o subespecies. No obstante, existen algunas morfotipos que sí corresponden a áreas geográficas específicas. Una de estas variedades fue descrita en 1868 por J. Anderson como L. anceps var. dawsonii, basándose en una planta recolectada en 1865 cerca de Santa Catarina Juquila, $241 \mathrm{~km}$ al suroeste de la ciudad de Oaxaca, México. Dicho taxón fue elevado al rango de especie en 1902 por Crawshay [L. dawsonii (J.Anderson) Crawshay, Gard. Chron. 32: 414. 1902] y en 1922 fue reconocido como una subespecie de L. anceps por Rolfe [L. anceps ssp. dawsonii (J.Anderson) Rolfe, Orch. Rev.30: 10. 1922].

Hasta ahora se reconocen dos subespecies de Laelia anceps con diferentes áreas de distribución geográfica: $L$. anceps subsp. anceps y L. anceps subsp. dawsonii. La subespecie dawsonii es relativamente escasa, con poblaciones aisladas que se distribuyen a lo largo de la Sierra Madre del Sur (Jalisco, Guerrero y Oaxaca; Figura 1) en la vertiente del Pacífico de México (Soto-Arenas, 1993). Actualmente se reconsidera volverla a tratar como una entidad específica distinta (G. Salazar, com. pers.) debido a las discontinuidades geográficas entre ambas subespecies y por algunas diferencias en sus atributos florales y fenológicos.

Laelia anceps subsp. anceps tiene una distribución geográfica más amplia y presenta poblaciones mucho más numerosas que las de la subespecie dawsonii (Soto-Arenas, 1993). La subespecie anceps se distribuye en la Sierra Madre Oriental, desde el estado de Nuevo León hasta el norte de Guatemala, incluyendo Tamaulipas, San Luis Potosí, Guanajuato, Querétaro, Hidalgo, Veracruz, Puebla, Oaxaca y Chiapas (Ames y Correll, 1985; Halbinger y Soto, 1997). En el estado de Veracruz es particularmente abundante, y de esta región es de donde provienen muchas de las variedades que se cultivan actualmente; en contraste, las poblaciones ubicadas al sur del Istmo de Tehuantepec son más bien escasas y poco estudiadas (Soto-Arenas, 1993). Aparentemen-

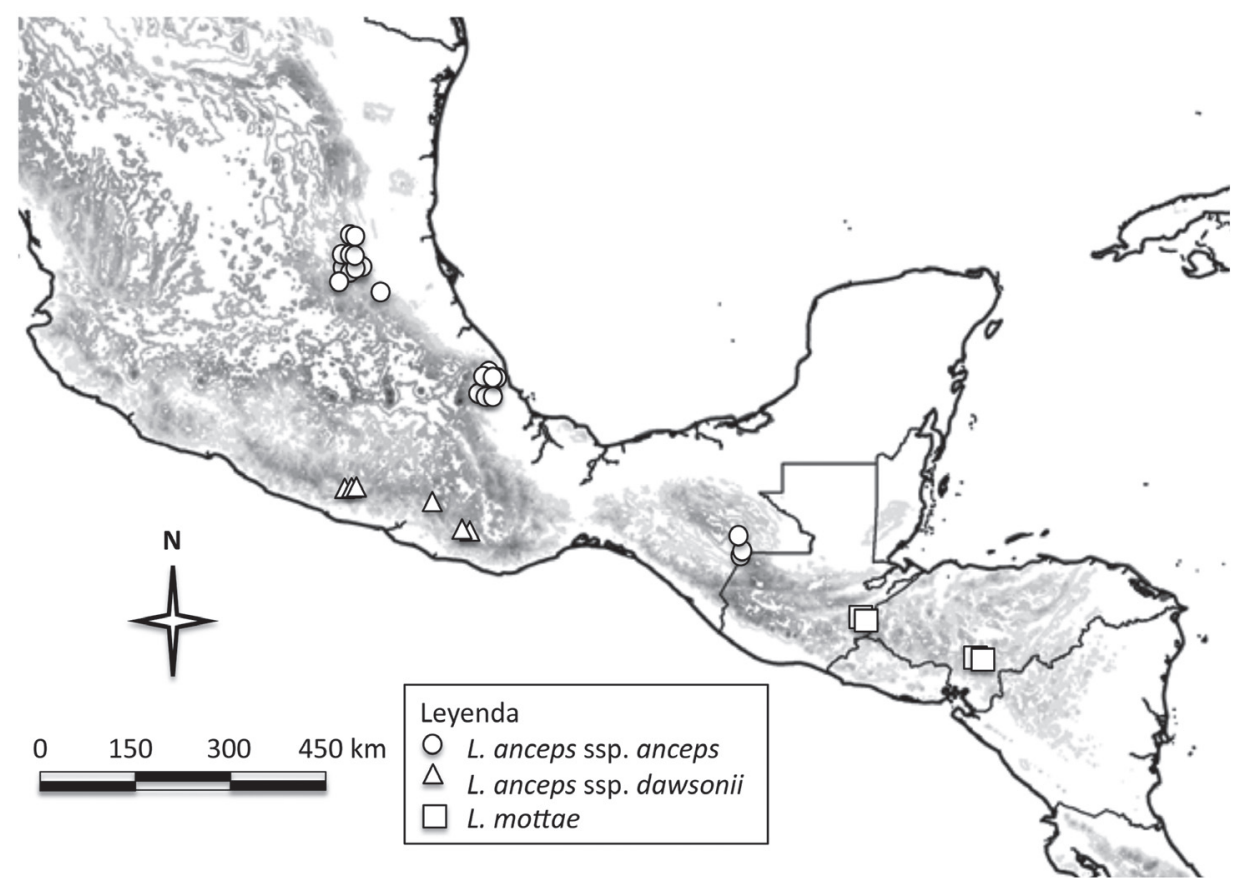

Figura 1. Distribución de los taxones del complejo de Laelia anceps tratados en este trabajo. 
te, las plantas de L.anceps subsp. anceps de Chiapas y del norte de Guatemala (Figura 1) tienen algunas diferencias con las de las regiones centrales y norteñas de México. Una de las diferencias es el color de las flores, ya que tienden a presentar tonos más claros que los de la forma típica. Es necesario un estudio más detallado para analizar estas diferencias y ver si es necesaria una reclasificación de las plantas de Chiapas y del norte de Guatemala en otra subespecie, dado que presentan una clara separación geográfica con las dos subespecies reconocidas hasta ahora.

En el sureste de Guatemala y en el sur de Honduras (Williams, 1952) se encuentran algunas poblaciones aisladas de lo que se hasta ahora se ha considerado como una variante enana de Laelia anceps (Halbinger y Soto, 1997). Sin embargo, estas poblaciones no se conocían bien y no se habían realizado estudios detallados de la morfología de estas plantas. Recientemente, el primer autor localizó varias poblaciones en el área de Esquipulas, departamento de Chiquimula, en Guatemala. Este hallazgo permitió observar que las plantas de la zona presentaban flores más pequeñas, inflorescencias 1(-2) floras, así como otros caracteres florales y vegetativos que difieren de los de las típicas L. anceps; y por lo tanto, se piensa que deben considerarse como una entidad diferente, que en este trabajo se propone como especie nueva para la ciencia.

En este trabajo se analizaron los ejemplares tanto de Laelia anceps como de L. mottae depositados en los herbarios Paul C. Standley (EAP) de Honduras, de la Universidad de San Carlos (BIGU) en Guatemala y del Herbario Nacional de México (MEXU), así como plantas cultivadas en varias colecciones particulares de Guatemala, Honduras y México. Además se revisó el acervo fotográfico de los herbarios EAP, MEXU (unibio.unam.mx, consultado 20 enero 2012) y MOBOT (Missouri Botanical Garden, www.tropicos.org, consultado 12 marzo 2013). Los ejemplares examinados se cotejaron con las descripciones de las especies del género Laelia, particularmente con los de L. anceps subsp. anceps (Soto-Arenas, 1993; Halbinger y Soto, 1997) y de L.anceps subsp. dawsonii forma dawsonii (Soto, 2002) y forma chilapensis (Soto, 2008).

Considerando que no existe algún nombre válido para las plantas del sur de Guatemala y de Honduras se propone el siguiente: Laelia mottae Archila, Chiron, Szlach. \& E.A. Pérez-García.

\section{Tratamiento taxonómico}

Laelia mottae Archila, Chiron, Szlach. \& E.A. Pérez-García sp. nov. (Figura 2).

Herba, Laeliae anceps Lindley similis, sed differt statura minore, inflorescentiis unifloris vel bifloris, labelli lobulo medio breviore, sepalo superiore oblongo, petalis rhombicis et labello trilobato; lobis lateralibus subtriangularibus, ad apicem rotundatis; lobo apicali oblongo ad apicem emarginato; ovario lineari $3.6 \mathrm{~cm}$ longo, $0.36 \mathrm{~cm}$ diametro; columna recurvata, ad apicem capitata. In Guatemala et Honduras

HOLOTIPO: Guatemala, departamento de Chiquimula, Esquipulas, alt. ca. $400 \mathrm{~m}$, bosque tropical caducifolio, septiembre 2010, F. Archila, FA-s.n. (BIGU). PARATIPO: Guatemala, departamento de Chiquimula, Esquipulas, cultivado por Javier Motta en la ciudad de Guatemala, alt. 388 m, agosto 2008, F. Archila, FA-s.n. (BIGU).

Plantas de 12 a $15 \mathrm{~cm}$ de altura sin incluir la inflorescencia, con pseudobulbos elípticos de $2.8-5.5 \mathrm{~cm}$ de $\operatorname{largo} \times 3 \mathrm{~cm}$ de ancho, elíptico-romboides en corte transversal, con 2 entrenudos, el basal de $1.3 \mathrm{~cm}$ de largo $\times 1.7 \mathrm{~cm}$ de ancho, el apical de $4.2 \mathrm{~cm}$ de largo $\times 3 \mathrm{~cm}$ de ancho; la región rizomatosa del tallo de $2.8 \mathrm{~cm}$ de largo $\times$ 0.4-0.7 cm de diámetro. Raíces cortas y numerosas de $0.2 \mathrm{~cm}$ de diámetro. Hojas elípticas, de $10-16 \mathrm{~cm}$ de largo $\times 2.5-3 \mathrm{~cm}$ de ancho, $0.2 \mathrm{~cm}$ de grueso. Inflorescencia de $20-30 \mathrm{~cm}$ de largo, normalmente uniflora, muy rara vez biflora; sépalos de color lavanda, el posterior oblongo con el ápice redondeado-aristado, de $3.25 \mathrm{~cm}$ de largo $\times 0.9 \mathrm{~cm}$ de ancho, los laterales de color lila claro, oblongo-elípticos con los ápices oblicuamente aristados, de $3.2 \mathrm{~cm}$ de largo $\times 0.82 \mathrm{~cm}$ de ancho; pétalos de color lila claro, rómbicos, apicalmente emarginados, de $3.35 \mathrm{~cm}$ de largo $\times 2.1 \mathrm{~cm}$ de ancho; labelo trilobulado, los lóbulos laterales subtriangulares, redondeados en el ápice, de $2.17 \mathrm{~cm}$ de largo $\times 0.88 \mathrm{~cm}$ de ancho; el lóbulo apical oblongo con el ápice entero o emarginado, de $1.15 \mathrm{~cm}$ de largo $\times 0.8 \mathrm{~cm}$ de ancho, con una mancha de color lila oscuro. Ovario pedicelado linear, de $3.6 \mathrm{~cm}$ de $\operatorname{largo} \times 0.36 \mathrm{~cm}$ de diámetro; columna de $2.26 \mathrm{~cm}$ de largo, recurvada, capitada en el ápice; clinandrio con tres protuberancias apicales; rostelo triangular (Figura 2).

Reconocimiento. Las plantas de Laelia mottae son, en general, más pequeñas que las típicas de $L$. anceps (Figura 3, Cuadro 1). Los pseudobulbos de L. mottae miden menos de seis centímetros de largo, en tanto que en las diferentes variedades de $L$. anceps su tamaño es mayor $(>6 \mathrm{~cm})$. También presentan inflorescencias más cortas, de menos de $40 \mathrm{~cm}$, que las de L. anceps, en las cuales llegan a medir más de $70 \mathrm{~cm}$. En L. mottae es muy común observar inflorescencias unifloras y en menor proporción bifloras, aún en plantas bien cultivadas. Al parecer las plantas de Honduras pueden llegar a producir más flores en cultivo (Battiti, com. pers.). En contraste, la floración típica de una L. anceps bien desarrollada es de dos a tres flores, y menos frecuentemente cuatro o más. Uno de los rasgos más distintivos de L. mottae es que los pétalos son relativamente anchos, algo similares a los que presenta L. anceps subsp. dawsonii. Cabe mencionar que las plantas de Guatemala y Honduras observadas no corresponden a esta última subespecie, ya que no coinciden 

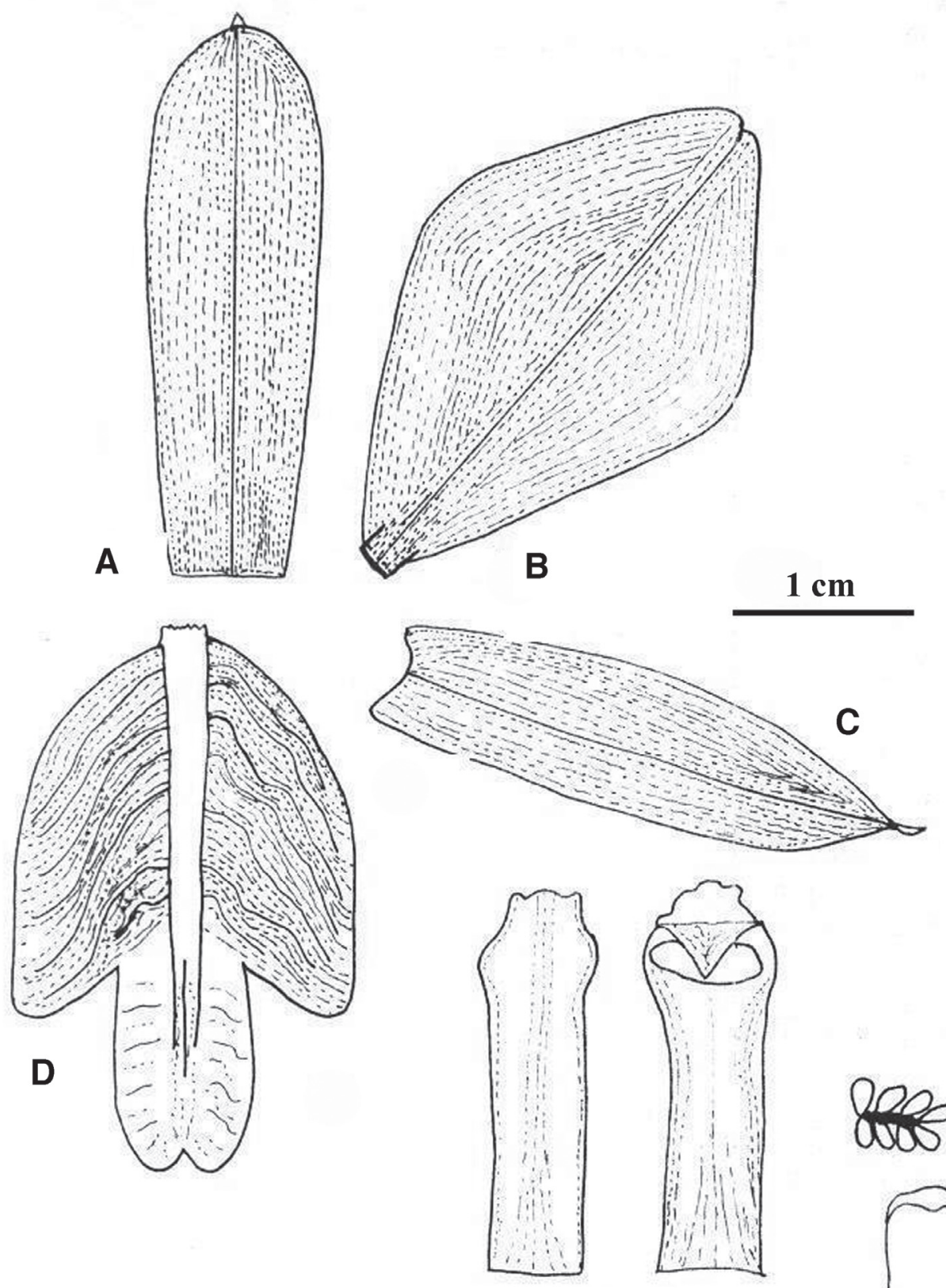

$\mathbf{E}$
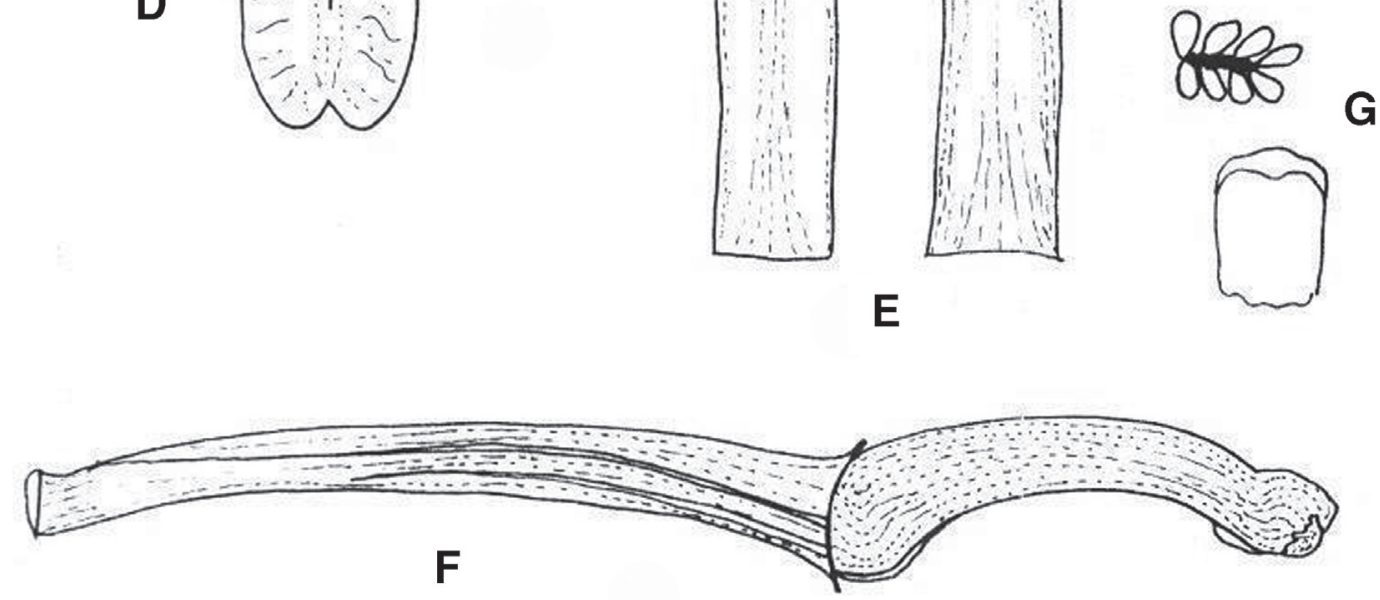

Figura 2. Esquema de Laelia mottae Archila, Chiron, Szlach. \& E.A. Pérez-García. (A) sépalo superior, (B) pétalo, (C) sépalo lateral, (D) labelo, (E) columna, (F) ovario y columna vistos lateralmente, $(\mathrm{G})$ antera y polinias. Dibujo de Fredy Archila.

en color, tamaño y forma de las flores, así como tampoco en su fenología reproductiva. Aunado a estas diferencias, estas especies tienen una distribución geográfica notablemente disyunta (Cuadro 1, Figura 1).
En la mayoría de las plantas de Laelia mottae el labelo presenta los lóbulos laterales planos (i.e. no reflexos), mientras que en L. anceps éstos generalmente son reflexos. En algunos ejemplares, entre ellos el tipo, el labelo no envuelve comple- 


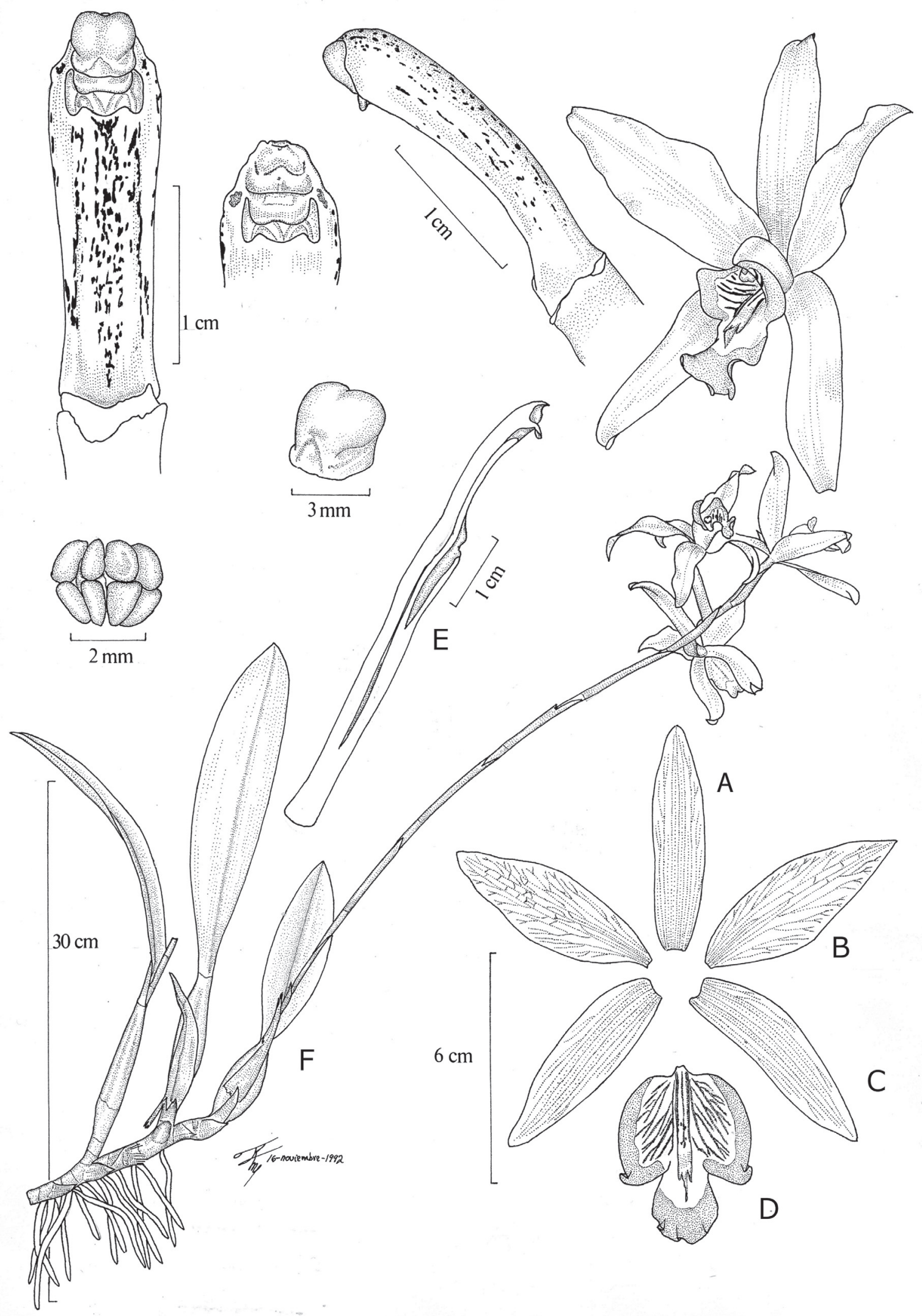

Figura 3. Esquema de Laelia anceps Lindl. subsp. anceps. (A) sépalo superior, (B) pétalo, (C) sépalo lateral, (D) labelo, (E) columna, (F) planta completa. Dibujo de Rolando Jiménez Machorro. 


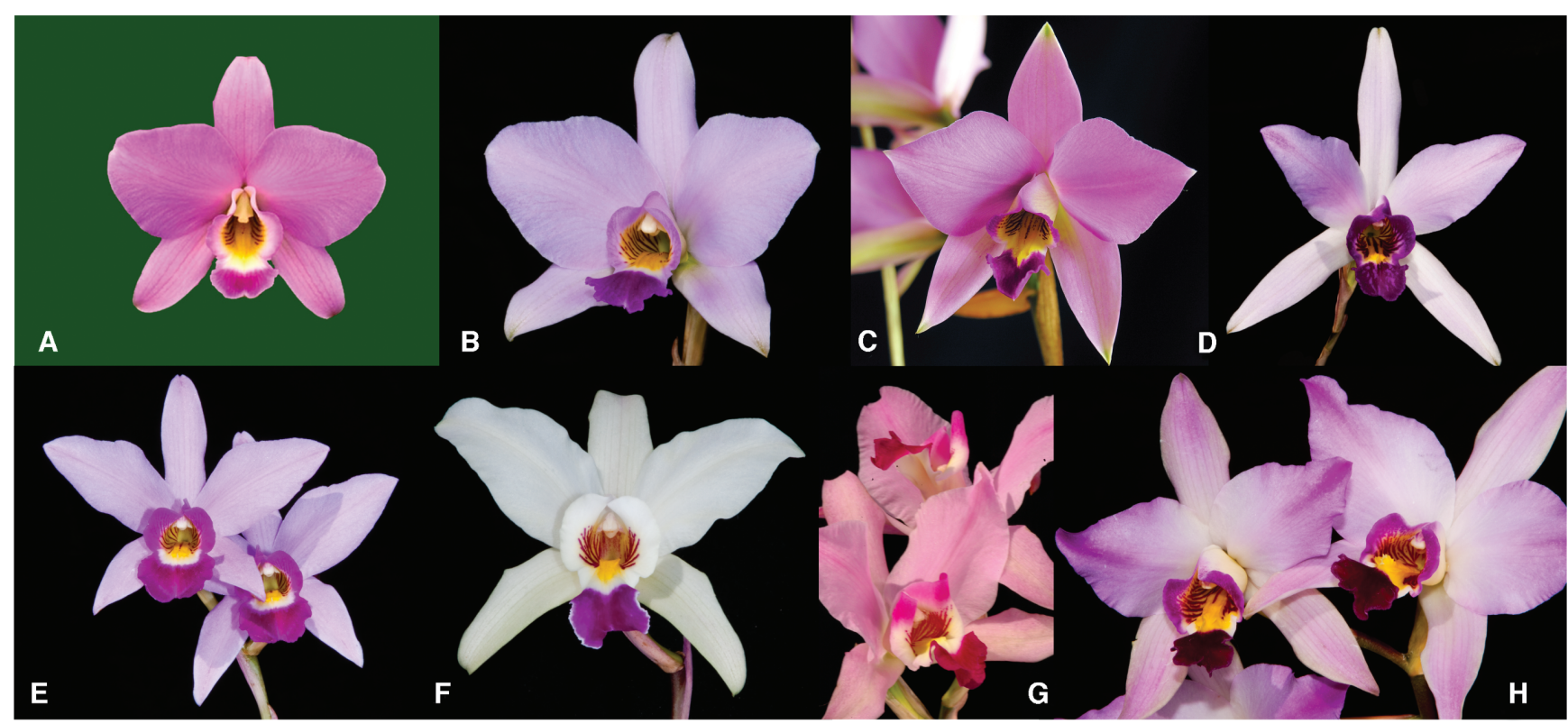

Figura 4. Comparativo de los taxones del complejo de Laelia anceps. L. mottae: (A) fotografía del ejemplar tipo, de Guatemala (foto Fredy Archila); (B) planta de la colección de F. Halbinger, probablemente de Guatemala, cultivada por E.A. Pérez-García (EAP-G) s.n. en la ciudad de México (foto EAP-G); (C) planta de Honduras, cultivada por Martín Battiti (foto M. Battiti). L. anceps subsp. anceps: (D) planta cultivada EAP-G 183, originaria de Chiapas, México (foto EAP-G); (E) ejemplar proveniente de la colección de F. Halbinger, cultivado EAP-G s.n, probablemente del estado de Veracruz, México (foto EAP-G). L. anceps subsp. dawsonii fo. dawsonii "Pacífico" (F) planta cultivada proveniente de la colección de F. Halbinger, EAP-G s.n., del estado de Oaxaca, México (foto EAP-G). L. anceps subsp. dawsonii (G) M.A. Soto 7449 (AMO) cultivada, individuo con características intermedias de color entre las formas dawsonii y chilapensis, proviene del estado de Oaxaca, México (foto EAP-G). L. anceps subsp. dawsonii forma chilapensis "Estrella del Sur" (H), ejemplar proveniente de la colección de F. Halbinger, cultivada EAP-G s.n., probablemente del estado de Guerrero, México (foto EAP-G). Nota: las fotografías no están a la misma escala.

tamente a la columna, lo cual permite que éste sea visible (Figura 4). En contraste, en las flores de L. anceps el labelo envuelve a la columna y por lo tanto ésta no se encuentra expuesta. Pero quizás el rasgo más distintivo de L. mottae sea que el labelo es notoriamente más corto en relación con los de las dos subespecies de L. anceps (Cuadro 1).

Distribución y ecología. En Guatemala, Laelia mottae ocupa un hábitat más cálido que el registrado para L. anceps, ya que se le encuentra a altitudes relativamente bajas, entre 400 y $450 \mathrm{~m}$, mientas que el intervalo altitudinal de L.anceps va de 1,300 a 1,600 m para la subsp. dawsonii (Soto 2002, 2008) y de 800 a 1,500 m para la subsp. anceps (Soto-Arenas, 1993). En la república de Honduras, L. mottae se ha tornado muy escasa, particularmente en las montañas de los alrededores de Tegucigalpa, en donde había una población relativamente grande pero aparentemente ya no existe (M. Battiti y A. Chinchilla, com. pers.). En esa área, L. mottae crecía entre 1,000 y $1,300 \mathrm{~m}$ de altitud, en bosques muy abiertos y secos, en suelos derivados de tobas volcánicas, creciendo como epífita y escasamente como litófita (A. Chinchilla, com. pers.). La temperatura promedio anual de la ciudad de Tegucigalpa (990 m s.n.m.) es de $22{ }^{\circ} \mathrm{C}$, y la precipitación media anual es de $880 \mathrm{~mm}$, la cual se concentra entre mayo y octubre. Las localidades exactas de L. mottae se omiten para evitar su recolección, pero las de Guatemala pueden solicitarse al primer autor. En la localidad tipo de esta especie en Guatemala, la precipitación media anual es de $855 \mathrm{~mm}$, pero las lluvias se presentan principalmente de junio a octubre. En Guatemala, L. mottae crece como epífita en bosques tropicales caducifolios (selvas bajas caducifolias o bosques estacionalmente secos). Algunas especies presentes en la zona son: Albizia caribaea (Urb.) Britton \& Rose, Alvaradoa amorphoides Liemb., Ceiba aesculifolia (Kunth) Britten \& Baker f., Cochlospermum vitifolium (Willd.) Spreng., Leucaena guatemalensis Britton \& Rose, Phyllocarpus septentrionalis Donn.Sm., Sabal mexicana Mart. y Swietenia humilis Zucc. El tipo de vegetación en el que se desarrolla L. mottae en Guatemala difiere del de L. anceps, ya que la última habita principalmente en bosques de encinos secos y húmedos, o incluso bosques mixtos de encino-pino (Quercus-Pinus), y raras veces en bosques mesófilos de montaña o en cafetales derivados de éstos. Al parecer L. mottae no coexiste con ninguna otra especie del mismo género, y quizá por ello no se conocen híbridos naturales de este taxón.

En cultivo, las plantas Laelia mottae requieren más calor y humedad que las de L. anceps, pero a ambas les favorecen los medios de cultivo muy aireados, de preferencia tablas o cestos de madera. Hasta ahora no se conocen muchos ejemplares de L. mottae en cultivo, y por lo tanto no 
Cuadro 1. Compartido de las características morfológicas y ecológicas entre Laelia anceps Lindl. subsp. anceps, L. anceps Lindl. subsp. dawsonii (J.Anderson) Rolfe (Soto, 2002; 2008) y L. mottae Archila, Chiron, Szlachetko \& E.A.Pérez-García.

\begin{tabular}{|c|c|c|}
\hline Laelia anceps subsp. anceps & Laelia anceps subsp. dawsonii & Laelia mottae \\
\hline $\begin{array}{l}\text { Inflorescencia (1)2-3(5)-florada, } \\
\text { hasta } 70 \mathrm{~m} \text { de largo. }\end{array}$ & $\begin{array}{l}\text { En la forma dawsonii, inflorescencia (1)2-3(4)-florada, } \\
\text { de } 40-70 \mathrm{~cm} \text { de largo; en la forma chilapensis, } \\
\text { inflorescencia (1)2-4(5)-florada, de } 40-60 \mathrm{~cm} \text { de largo. }\end{array}$ & $\begin{array}{l}\text { Inflorescencia 1-2 (3)-florada, } \\
\text { hasta } 30 \mathrm{~cm} \text { de largo. }\end{array}$ \\
\hline $\begin{array}{l}\text { Flores de } 7-12 \mathrm{~cm} \text { de alto } \times 5.5-8 \mathrm{~cm} \\
\text { de ancho. }\end{array}$ & $\begin{array}{l}\text { Flores: en la forma dawsonii, de } 7.3-12 \mathrm{~cm} \text { de alto } \times \\
\text { 5.5-8 cm de ancho; en la forma chilapensis, de } \\
8.5-12 \mathrm{~cm} \text { de alto } \times 7-9.5 \mathrm{~cm} \text { de ancho. }\end{array}$ & $\begin{array}{l}\text { Flores de ca. } 5.4 \mathrm{~cm} \text { de alto } \times \text { ca. } \\
5.9 \mathrm{~cm} \text { de ancho. }\end{array}$ \\
\hline $\begin{array}{l}\text { Pétalos elípticos con ápice agudo, } \\
\text { de 49-69 mm largo } \times 17-27 \mathrm{~mm} \text { de ancho }\end{array}$ & $\begin{array}{l}\text { Pétalos rómbicos con el ápice agudo, de 38-65 mm } \\
\text { largo } \times 19-33 \mathrm{~mm} \text { de ancho. }\end{array}$ & $\begin{array}{l}\text { Pétalos rómbicos con el ápice redon- } \\
\text { deado o emarginado, de ca. } 33.5 \mathrm{~mm} \\
\text { de largo } \times \text { ca. } 21 \mathrm{~mm} \text { de ancho. }\end{array}$ \\
\hline
\end{tabular}

Sépalos y pétalos rosados o lila, normalmente ambos en el mismo tono. En algunas plantas de Chiapas y del norte de Guatemala los sépalos son más claros que los pétalos.

Sépalo dorsal de 47-63 mm de largo $\times$ 9-14 cm de ancho, y los sépalos laterales de 44-61 mm de largo $\times$ 9-15 mm de ancho.

Labelo: de 38-47 mm de largo × 31-40 mm de ancho.

Labelo rosado con ápice de los tres lóbulos púrpura o lila intenso.

Lóbulo central del labelo oblongo, con el ápice algo o marcadamente deflexo. Con tres quillas carnosas.

Labelo trilobulado, con los lóbulos laterales marcadamente reflexos.

Pseudobulbos de 6-10 cm de largo $x$ $1.2-2.1 \mathrm{~cm}$ de ancho

Florecen de octubre a diciembre; las plantas de Chiapas y del norte de Guatemala florecen desde finales de agosto a septiembre.

De 800 a 1,500 m de altitud.

Distribución: Sierra Madre Oriental (Tamaulipas, San Luis Potosí, Hidalgo, Puebla y Veracruz), Sierra Norte de Oaxaca y montañas del Altiplano Mexicano (Guanajuato y Querétaro), Macizo Central de Chiapas y norte de Guatemala.
En la forma dawsonii sépalos y pétalos blancos, muy raras veces ligeramente rosados.

En la forma chilapensis sépalos rosados (más claros que los pétalos), pétalos lila con el ápice más oscuro.

En la forma dawsonii el sépalo dorsal de 45-69 mm de largo $\times 10-18 \mathrm{~mm}$ de ancho y los sépalos laterales de 45-60 mm de largo $\times 14-16 \mathrm{~mm}$ de ancho.

En la forma chilapensis el sépalo dorsal de 42-62 mm cm de largo $\times 13-18 \mathrm{~mm}$ de ancho, y los sépalos laterales de 39-50 mm de largo × 12-17 mm de ancho.

Labelo: en la forma dawsonii de 38-50 mm de largo $\times 34-45 \mathrm{~mm}$ de ancho; en la forma chilapensis de 43-51 mm de largo $\times$ $32-41 \mathrm{~mm}$ de ancho.

En la forma dawsonii, con los ápices de los lóbulos laterales de labelo blancos, el lóbulo medio blanco o con una mancha lila en el lóbulo medio, en algunos cultivares el lóbulo medio es de color lila oscuro. En la forma chilapensis, labelo rosado con los ápices de los lóbulos laterales de color lila, y el lóbulo medio de color púrpura intenso, aterciopelado.

Lóbulo central del labelo ovobado a suborbicular. En la forma dawsonii tiende a estar marcadamente deflexo; en la forma chilapensis sólo es algo deflexo. En ambas fromas con 3 a 5 quillas carnosas.

Labelo trilobulado, con los lóbulos laterales marcadamente reflexos.
Sépalos y pétalos lila o lila-cerúleo, normalmente ambos en el mismo tono.

Sépalo dorsal de ca. $32.5 \mathrm{~mm}$ de largo $\times$ ca. $9 \mathrm{~mm}$ de ancho, y los sépalos laterales de ca. $32 \mathrm{~mm}$ de largo $\times$ $8.2 \mathrm{~mm}$ de ancho.

Labelo: de ca. $21.7 \mathrm{~mm}$ de largo $\times$ ca. $8.8 \mathrm{~mm}$ de ancho.

Labelo lila-cerúleo y generalmente sólo el lóbulo medio presenta un lila más intenso. Algunas plantas de honduras presentan los ápices de los tres lóbulos de color más oscuro.

Lóbulo central del labelo oblongo, generalmente no deflexo. Con tres quillas carnosas.

Labelo trilobulado con lóbulos laterales planos. En algunos ejemplares, el labelo no envuelve completamente a la columna, permitiendo que el ginostemo sea visible por en medio de los lóbulos laterales.

Pseudobulbos de 8-11 cm de largo. Algunas plantas de Pseudobulbos de 2.8-5.5 cm de la forma dawsonii son incluso más grandes $(18 \mathrm{~cm})$, algo semejantes a $L$. superbiens, de $2.2-4 \mathrm{~cm}$ de ancho.

Floración: en la forma dawsonii de noviembre a enero; en la forma Chilapensis de finales de octubre a noviembre.

De 1,300-1,600 m de altitud.

Distribución: Sierra Madre del Sur (Oaxaca y Guerrero) y Sierra Madre Occidental (Jalisco). largo $\times$ ca. $3 \mathrm{~cm}$ de ancho. Algunas plantas de Honduras florecen en octubre.

En Guatemala de 400 a $450 \mathrm{~m}$ de altitud; en Honduras de 1,000 a 1,300 m de altitud.

Distribución: sureste de Guatemala y sur de Honduras
Florecen de agosto a septiembre. 
hay variedades reconocidas (o cultivares) para esta especie; particularmente no se conocen formas albas, semialbas o cerúleas. Existe un vivero en Santa Bárbara, California, que comercializa algunos cultivares de L. mottae bajo el nombre de "L. anceps de Guatemala" (http://sborchid.com/index. php), así como también venden algunas plantas que pueden ser híbridos logrados en vivero entre L. mottae y L. anceps. Esta es otra de las razones por las cuales $L$. mottae debe considerarse como una especie diferente de L. anceps, ya que este nivel taxonómico es más reconocido en la sociedad en general, respecto a alguna categoría infraespecífica. Con el estatus de especie se puede esperar que la integridad genética de este llamativo taxón centroamericano tenga mayores posibilidades de conservación.

Etimología. El nombre de la especie está dedicado a Javier Motta, gran cultivador y amante de las orquídeas.

Ejemplares adicionales examinados. Laelia mottae. GUATEMALA: F. Archila s.n. septiembre de 1995 (BIGU); E.A. Pérez-García s.n. ejemplar cultivado y fotografiado en la ciudad de México, proveniente de la colección de Federico Halbinger, probablemente de Guatemala (Foto A, Figura 4). HONDURAS: Louis O. Williams Eap-10019 (EAP), Colector desconocido BRAHMS 4564 (EAP); M. Mattiti s.n. ejemplar cultivado (foto C, Figura 4). Laelia anceps subsp. anceps. Hidalgo: F. González-Medrano 8438 (MEXU); Querétaro: L.M. Chávez 109 (MEXU), S. Zamudio 7015 (MEXU), H. Rubio 224 (MEXU), B. Servín Orozco 1389 (MEXU), L. López Ch. 744 (MEXU); San Luis Potosí: A. Carbajal E. s.n. (MEXU); Veracruz: R. Cedillo 317 (MEXU), G. Castillo y W. Busey 2989 (MEXU), M. Rosas 733 (MEXU), M. Rosas 678 (MEXU), H. Kruse 19731205281; Chiapas: $E A P-G 183$ (foto D, Figura 4). Laelia anceps subsp. dawsonii. Guerrero: H. Kruse 19731101-264 (MEXU), Hágsater 3680 (MEXU), O. Nagel 2670 MEXU, EAP-G s.n. (foto H, Figura 5); Oaxaca: E. Hunn OAX-517 (MEXU), I. Trujillo Olazo 1053 (MEXU), cultivar "Pacífico" EAP-G s.n. (foto F, Figura 4), M.A. Soto 7449 ejemplar cultivado (foto G, Figura 4).

\section{Agradecimientos}

Se agradece al personal del herbario Paul C. Standley (EAP), de la república de Honduras, por las fotografías de sus ejemplares de herbario. Rolando Jiménez Machorro proporcionó el dibujo de Laelia anceps. Marco A. Rome-
ro-Romero revisó y editó este manuscrito. Martín Battiti proporcionó fotografías y comentarios sobre las plantas de Honduras y Nelson Cancino aportó comentarios sobre la variabilidad de L. mottae. Fernado Chiang Cabrera realizó la diagnosis en latín. Adolfo Espejo y dos revisores anónimos aportaron valiosas sugerencias a las versiones previas de este manuscrito.

\section{Literatura citada}

Alrich P. y Higgins W. 2008. The Marie Selby Botanical Gardens. Illustrated Dictionary of Orchid Genera. Cornell University Press, Sarasota.

Ames O. y Correll D.S. 1985. Orchids of Guatemala and Belize. Dover Publications, Nueva York.

Chiron G.R. y Castro-Neto V.P. 2002. Révision des espèces brésiliennes du genre Laelia Lindley. Richardiana 2:4-28.

Halbinger F. y Soto M. 1997. Laelias of México. Orquídea (Méx.) 15: $1-160$

Lindley J. 1831. The Genera and Species of Orchidaceous Plants 96: 115 .

Salazar G.A., Reyes-Santiago J., Brachet C. y Pérez-Crisanto J. 2006. Orquídeas y otras Plantas Nativas de La Cañada, Cuicatlán, Oaxaca, México. Instituto de Biología, Universidad Nacional Autónoma de México, México, D.F.

Soto-Arenas M.A. 1993. Clasificación infraespecífica de Laelia anceps. Orquídea (Méx.) 13:125-144.

Soto M. 2002. Laelia anceps Lindl. subsp. dawsonii (J.Anderson) Rolfe f. dawsonii. En: Hágsater E. y Soto M. Eds. Icones Orchidacearum 5-6. Lámina 597. Herbario AMO, México, D.F.

Soto M. 2008. Laelia anceps subsp. dawsonii f. chilapensis Soto Arenas. En: Hágsater E. y Soto M. Eds. Icones Orchidacearum 10. Lámina 1035. Herbario AMO, México, D.F.

van den Berg C. y Chase M.W. 2000. Nomenclatural notes on Laeliinae - I. Lindleyana 15:115-119.

van den Berg C. y Chase M.W. 2001. Nomenclatural notes on Laeliinae - II. Additional combinations and notes. Lindleyana 16:109-112.

van den Berg C. y Chase M.W. 2004a. A chronological view of Laeliinae taxonomical history. Orchid Digest 68:226-230, 252254.

van den Berg C. y Chase M.W. 2004b. Nomenclatural notes on Laeliinae - IV. New combinations in Laelia and Sophronitis. Kew Bulletin 59:565-567.

van den Berg C., Higgins W.E., Dressler R.L., Whitten W.M., Soto-Arenas M.A. y Chase M.W. 2009. A phylogenetic study of Laeliinae (Orchidaceae) based on combined nuclear and plastid DNA sequences. Annals of Botany 104:417-430.

Williams L.O. 1952. Laelia anceps in Honduras. Orchid Journal 1:194. 